\title{
Identification of novel common breast cancer risk variants at the $6 q 25$ locus among Latinas
}

Joshua Hoffman', Laura Fejerman², Donglei Hư ${ }^{3}$, Scott Huntsman ${ }^{3}$, Min Li ${ }^{3}$, Esther M. John ${ }^{4,5}$, Gabriela Torres-Mejia ${ }^{6}$, Larry Kushi ${ }^{7}$, Yuan Chun Ding ${ }^{8}$, Jeffrey Weitzel ${ }^{9}$, Susan L. Neuhausen ${ }^{8}$, Paul Lott ${ }^{10}$, COLUMBUS Consortium, Magdalena Echeverry ${ }^{11}$, Luis Carvajal-Carmona ${ }^{10}$, Esteban Burchard ${ }^{12,13}$, Celeste Eng ${ }^{12,13}$, Jirong Long ${ }^{14}$, Wei Zheng ${ }^{14}$, Olufunmilayo Olopade ${ }^{15}$, Dezheng Huo ${ }^{16}$, Christopher Haiman ${ }^{17}$ and Elad Ziv ${ }^{2 *}$ (D)

\begin{abstract}
Background: Breast cancer is a partially heritable trait and genome-wide association studies (GWAS) have identified over 180 common genetic variants associated with breast cancer. We have previously performed breast cancer GWAS in Latinas and identified a strongly protective single nucleotide polymorphism (SNP) at 6q25, with the protective minor allele originating from indigenous American ancestry. Here we report on fine mapping of the $6 \mathrm{q} 25$ locus in an expanded sample of Latinas.

Methods: We performed GWAS in 2385 cases and 6416 controls who were either US Latinas or Mexican women. We replicated the top SNPs in 2412 cases and 1620 controls of US Latina, Mexican, and Colombian women. In addition, we validated the top novel variants in studies of African, Asian and European ancestry. In each dataset we used logistic regression models to test the association between SNPs and breast cancer risk and corrected for genetic ancestry using either principal components or genetic ancestry inferred from ancestry informative markers using a model-based approach.
\end{abstract}

Results: We identified a novel set of SNPs at the $6 \mathrm{q} 25$ locus associated with genome-wide levels of significance $\left(p=3.3 \times 10^{-8}-6.0 \times 10^{-9}\right)$ not in linkage disequilibrium (LD) with variants previously reported at this locus. These SNPs were in high LD $\left(r^{2}>0.9\right)$ with each other, with the top SNP, rs3778609, associated with breast cancer with an odds ratio (OR) and $95 \%$ confidence interval $(95 \% \mathrm{Cl})$ of $0.76(0.70-0.84)$. In a replication in women of Latin American origin, we also observed a consistent effect (OR 0.88; $95 \% \mathrm{Cl} 0.78-0$. 99; $p=0.037$ ). We also performed a meta-analysis of these SNPs in East Asians, African ancestry and European ancestry populations and also observed a consistent effect (rs3778609, OR 0.95; 95\% Cl 0.91-0.97; $p=0.0017$ ).

Conclusion: Our study adds to evidence about the importance of the 6q25 locus for breast cancer susceptibility. Our finding also highlights the utility of performing additional searches for genetic variants for breast cancer in non-European populations.

Keywords: Genome wide association study, Fine mapping, Hispanic/Latino populations

\footnotetext{
*Correspondence: Elad.Ziv@ucsf.edu; Elad.ziv@ucsf.edu

${ }^{2}$ Division of General Internal Medicine, Department of Medicine, Institute of

Human Genetics, Helen Diller Family Comprehensive Cancer Center,

University of California, San Francisco, Box 0320, San Francisco, CA 94143,

USA

Full list of author information is available at the end of the article
}

(c) The Author(s). 2019 Open Access This article is distributed under the terms of the Creative Commons Attribution 4.0 International License (http://creativecommons.org/licenses/by/4.0/), which permits unrestricted use, distribution, and reproduction in any medium, provided you give appropriate credit to the original author(s) and the source, provide a link to the Creative Commons license, and indicate if changes were made. The Creative Commons Public Domain Dedication waiver (http://creativecommons.org/publicdomain/zero/1.0/) applies to the data made available in this article, unless otherwise stated. 


\section{Introduction}

Breast cancer is a partially heritable disease. Mutations in several high-penetrance genes including BRCA1 [1, 2], BRCA2 [3], and others [4] are associated with high risk of breast cancer among carriers and explain a fraction of the heritability. Genome-wide association studies (GWAS) have identified over 180 common single nucleotide polymorphisms (SNPs) associated with risk of breast cancer [5-20]. The majority of these SNPs were identified in European ancestry and East Asian ancestry populations, although some unique SNPs have been identified in African American populations [21] and in Latina populations [22, 23].

Several GWAS studies have identified SNPs at $6 \mathrm{q} 25$ that are associated with breast cancer risk [13, 18, 20, 23-27] and mammographic density [23, 27-30]. The initial report identified a SNP in the intergenic region between ESR1 and CDCC170 in an East Asian population [24]. The locus was then confirmed in other populations and several additional variants were identified $[11,18$, 25, 26, 31]. More recently, a fine-mapping and functional approach at this locus identified five distinct common variants associated with risk of different subtypes of breast cancer [27].

Hispanic/Latino populations are the second largest ethnic group in the USA [32] and yet have been understudied in GWAS [33]. Latinos are a population of mixed ancestry with European, indigenous American and African ancestral contributions [34-37]. Since there are no large studies of breast cancer in indigenous American populations, studies in Latinos may identify novel variants associated with breast cancer that are unique to or substantially more common in this population. We have previously used an admixture mapping approach to search for breast cancer susceptibility loci in Latinas and identified a large region at 6q25 where indigenous American ancestry was associated with decreased risk of breast cancer [22]. Subsequently, we identified a SNP (rs140068132) that was common (minor allele frequency $\sim 0.1$ ) only in Latinas with indigenous American ancestry and was associated with substantially lower risk of breast cancer, particularly estrogen receptor (ER)-negative breast cancer, and with lower mammographic density [23]. However, the variant we identified did not completely explain the risk associated with locus-specific ancestry at 6q25 in Latinas, suggesting that other variants may account for this risk. We set out to fine-map and identify additional variants at 6 q25 associated with breast cancer risk among Latinas.

\section{Methods}

\section{Populations}

San Francisco Bay Area breast cancer study (SFBCS): the SFBCS is a population-based multiethnic case-control study of breast cancer. Patients (cases) aged 35-79 years diagnosed with invasive breast cancer from 1995 to 2002 were identified through the Greater Bay Area Cancer Registry. Controls were identified by random-digit dialing and matched on 5-year age groups. Blood collection was initiated in 1999. For this study, we focused only on patients and matched controls who self-identified as Latina or Hispanic and included 351 cases and 579 controls. Samples from this study were used as part of the initial discovery set.

Breast Cancer Family Registry (BCFR): the BCFR is an international, National Cancer Institute (NCI)-funded family study that has recruited and followed over 13,000 breast cancer families and individuals with breast cancer with strong likelihood of genetic contribution to disease. The present study includes samples from the population-based Northern California site of the BCFR. Cases in patients aged 18-64 years diagnosed from 1995 to 2007 were ascertained through the Greater Bay Area Cancer Registry. Cases in patients with indicators of increased genetic susceptibility (diagnosis at the age of $<35$ years, bilateral breast cancer with the first diagnosis at the age of $<50$ years, a personal history of ovarian or childhood cancer, and a family history of breast or ovarian cancer in first-degree relatives) were oversampled. Cases not meeting these criteria were randomly sampled.

Population controls were identified through random-digit dialing and frequency-matched on 5-year age groups to cases diagnosed from 1995 to 1998 . We included 641 cases and 61 controls who self-identified as Latina or Hispanic from this study. Samples from this study were used as part of the initial discovery set.

Since the SFBCS and BCFR were recruited from the same region and during an overlapping time frame, we combined these datasets to search for relatives. After removing relatives (preferentially keeping cases) and samples that overlapped with the Kaiser Research Project on Genes, Environment and Health, we included 942 cases and 589 controls from these studies.

Multiethnic cohort (MEC): the MEC is a large prospective cohort study in California (mainly Los Angeles County) and Hawaii. The breast cancer study is a nested case-control study including women with invasive breast cancer diagnosed at the age of $>45$ years and controls matched on age (within 5 years) and self-identified ethnicity. After removing relatives (preferentially keeping cases), we used phenotypic and genetic data from 520 Latina breast cancer cases and 1544 matched Latina controls. Samples from this study were used as part of the initial discovery set.

Research project on genes environment and health (RPGEH): the RPGEH is a large cohort study of over 100,000 men and women of all racial/ethnic groups who are members of the Kaiser Permanente Health Plan. This 
analysis focuses only on women who are of self-reported Latina/Hispanic ethnicity $(N=3801)$. We included both incident and prevalent cases (total $N=225$ ) in our analyses. We identified 44 women who were also included in the SFBCS. The genetic data from these participants were included as part of the RPGEH since we considered the Affymetrix Lat array as a more comprehensive array than the Affymetrix 6.0 array. After removing relatives, we included a total of 225 cases and 3574 controls. Samples from this study were used as part of the initial discovery set.

Cancer de mama (CAMA) study: this study is a population-based case-control study of breast cancer conducted in Mexico City, Monterrey, and Veracruz. Patients (cases) aged 35-69 years diagnosed between 2005 and 2007 were recruited from 11 hospitals (3-5 in each region). Controls were recruited based on membership in the same health plan as the cases and are frequency-matched on 5 -year age groups. For the current study, we used phenotypic data and DNA samples from 1008 women with breast cancer and 1063 controls. Of these, 698 cases and 599 controls were genotyped with the Illumina Oncoarray and included in the discovery. An additional 310 cases and 464 controls were included as part of the replication dataset.

Colombian Study of Environmental and Heritable Causes of Breast Cancer (COLUMBUS): COLUMBUS is a population-based case-control study of breast cancer conducted in four cities: Bogota, Ibague and Neiva from the Central Colombian Andes region, and Pasto, from the Colombian South. Patients aged 18-75 years, with incident cases of invasive breast cancer, have been recruited in two population registries and two large cancer hospitals. Recruitment started in 2011. Cancer-free controls were recruited through the same institutions and were matched on education, socioeconomic status and local origin using a genealogical interview. In the current study, we used data from 954 cases and 769 controls for the replication study.

Hereditary Cancer Registry of City of Hope $(\mathrm{HCRCOH})$ (Southern California; PI Jeffrey Weitzel): Latina breast cancer cases are part of the $\mathrm{HCRCOH}$ through the Clinical Cancer Genetics Community Research Network (CCGCRN). The CCGCRN includes cancer center and community-based clinics that provide genetic counseling to individuals with a personal or family history of cancer [38]. All patients are invited to participate in the $\mathrm{HCRCOH}$ at the time of consultation (> 90\% participation). Starting in May 1998 and continuing to the present, women of self-reported Latina origin with breast cancer were seen for genetic counseling, were enrolled in the Registry and underwent $B R C A 1 / 2$ testing after providing informed consent. In the current study we genotyped 1148 cases. The 347 unaffected female Latina controls were from Southern California and were invited to participate at community health fairs, via flyers, and at City of Hope. These samples were used as part of the replication study.

African American breast cancer GWAS (AABC): the GWAS includes African American participants from nine epidemiological studies of breast cancer, comprising a total of 3153 cases and 2831 controls (cases/controls: the MEC, 734/1003; the Los Angeles component of the Women's contraceptive and reproductive experiences (CARE) study, 380/224; the Women's circle of health study (WCHS), 272/ 240; the SFBCS, 172/231; the Northern California Breast Cancer Family Registry (NC-BCFR), 440/53; the Carolina breast cancer study (CBCS), 656/608; The Prostate, Lung, Colorectal, and Ovarian Cancer Screening Trial (PLCO) Cohort, 64/133; the Nashville breast health study (NBHS), 310/186; and the Wake Forest University breast cancer study (WFBC), 125/153). Additional details have previously been reported [21,39]. These samples were used as part of the replication study.

The ROOT consortium included six studies and a total of 1657 cases and 2029 controls of African ancestry: the Nigerian Breast Cancer Study (NBCS), 711/624; the Barbados national cancer study (BNCS), 92/229; the Racial variability in genotypic determinants of breast cancer risk study (RVGBC), 145/257; the Baltimore Breast cancer study (BBCS), 95/102; the Chicago cancer prone study (CCPS), 394/387; and the Southern community cohort (SCCS), 220/430. Additional details can be found elsewhere [21]. These samples were used as part of the replication study.

Shanghai breast cancer genetics study: study participants were drawn from four population-based studies conducted in Shanghai, the Shanghai Breast Cancer Study (SBCS), Shanghai Women's Health Study (SWHS), Shanghai Breast Cancer Survival Study (SBCSS), and the Shanghai Endometrial Cancer Study (SECS (which contributed control data only). The SBCS is a population-based, case-control study conducted in urban Shanghai. Subject recruitment in the initial phase of the SBCS (SBCS-I) was conducted between August 1996 and March 1998. The second phase (SBCS-II) of recruitment occurred between April 2002 and February 2005. Breast cancer cases were identified through the population-based Shanghai Cancer Registry and supplemented by a rapid case-ascertainment system. Controls were randomly selected using the Shanghai Resident Registry. The SBCSS included newly diagnosed breast cancer cases ascertained via the Shanghai Cancer Registry between April 2002 and December 2006. The SECS is a population-based, case-control study of endometrial cancer conducted between January 1997 and December 2003 using a protocol similar to the SBCS; only community controls from the SECS were included in the present study. The SWHS is a population-based prospective cohort study of women recruited between 1996 and 
2000. The cohort has been followed by a combination of record linkage and active follow up to identify cause-specific mortality and cancer incidence by sites. All these studies are conducted among Chinese women in Shanghai, using very similar protocols in data and sample collection. There were 2731 cases and 2135 controls genotyped with an Affymetrix 6.0 array and 1794 cases and 2059 controls genotyped with an Illumina MEGA array. These subsets were analyzed separately and included in a meta-analysis as part of the replication study.

European ancestry GWAS data: we also evaluated the top SNPs using summary statistics from a recent large GWAS of European-ancestry breast cancer cases and controls [40]. We downloaded the summary statistics the Breast Cancer Association Consortium (BCAC) website (http://bcac.ccge.medschl.cam.ac.uk/bcacdata/oncoarray/) and used the summary statistics from the combined analysis of individuals of European ancestry from the Oncoarray and iCOGS consortia.

\section{Genotyping GWAS}

The SFBCS and NC-BCFR samples were all genotyped with an Affymetrix 6.0 arrays at the University of California, San Francisco (UCSF). The MEC samples were genotyped with an Illumina 660 array at USC (520 Latina women with breast cancer and 546 matched Latina controls) and an additional 998 controls were typed on an Illumina $2.5 \mathrm{M}$ array at the Broad Institute (Cambridge, MA, USA). The RPGEH samples were typed on an Affymetrix LAT array at UCSF. The CAMA samples were typed on an Ilumina Oncoarray at the Quebec Genome Center. The COLUMBUS samples were typed on an Affymetrix Biobank Array. Genotyping in the AABC consortium was conducted using the IlluminaHuman1M-Duo BeadChip. Genotyping in the ROOT consortium was conducted using the Illumina HumanOmni2.5-8v1 array at Johns Hopkins University Center for Inherited Disease Research. A subset of the Shanghai Breast Cancer Genetics Study (SBCGS) samples were typed on an Affymetrix 6.0 array. After quality control exclusions, the final data set included 2731 cases and 2135 controls. A second subset of the SBCGS were genotyped on an Illumina MEGA array. After quality control exclusions, the final data set included 1794 cases and 2059 controls. Data for four SNPs identified in the discovery stage were extracted from the SBCGS datasets and were included in the replication stage.

\section{Replication genotyping}

The CAMA samples and the CCGCRN samples, were genotyped using Taqman probes for rs3778609. The CAMA samples that were not included in the GWAS were genotyped at 106 ancestry informative markers from genotyped on a Sequenome platform as previously described [41]. CCGCRN samples included 100 ancestry informative markers that were included as part of a sequencing project. The sequence data were aligned to Human Genome Build 37 using Burrows-Wheeler alignment and genotype calls were made using Haplotypecaller, which is part of the GATK platform [42].

\section{Analysis \\ Genotyping quality control and imputation}

Samples with $>5 \%$ missing genotypes were removed from each dataset. We dropped variants with $>5 \%$ missing data from each dataset. Since excess homozygosity is more common in populations with substructure, particularly with ancestry informative markers, we did not use deviation from Hardy-Weinberg equilibrium as a criterion for excluding markers. All datasets were entered mapped to Hg19. Each dataset was then phased using SHAPEIT and imputed using the Haplotype Reference Consortium (HRC) with Minimac3 [43]. For the MEC datasets that included both $660 \mathrm{~K}$ and $2.5 \mathrm{M}$ arrays, we used the overlapping SNPs $(N=192,795)$ and imputed from those since we found that if imputing them separately and then analyzing them together produced a large number of false positives. Each of the remaining GWAS datasets were submitted to the HRC server individually for imputation. Only variants with imputation quality scores of $R^{2}>0.5$ were selected for additional analysis. In a separate analysis, we imputed each of the datasets to the 1000 Genomes Reference Version 3 (October 2015 release) [44] with Minimac3.

Genotype imputation for the ROOT consortium was conducted using the IMPUTE2 software [45] with the 1000 Genomes Project phase I cosmopolitan variant set as the reference panel (October 2011 release) [43]. Genotype imputation in $\mathrm{AABC}$ was conducted using IMPUTE2 software [45] to a cosmopolitan panel of all 1000 Genome Project subjects (March 2012 release). Variants with imputation score $>0.3$ were included in the analysis.

The Shanghai Breast Cancer Study GWAS data were phased with Minimac2 and imputed with SHAPEIT using 1000 Genomes Project phase 3. Only SNPs with a minor allele frequency (MAF) $\geq 0.01$ and high imputation quality $(\mathrm{RSQR} \geq 0.5)$ were included in the analyses.

We used KING [46] to identify relative pairs either within the RPGEH cohort or between the RPGEH and SFBCS and/or NC-BCFR and performed the same analysis within the MEC and the CAMA study. We identified pairs of individuals with kinship coefficient $>0.2$ and dropped one from each of these pairs. If a relative pair included a case and control then we excluded the control. If a relative pair included two cases or two controls we randomly dropped one of them. We dropped 127 individuals to eliminate all closely related individuals from the combined RPGEH, SFBCS, and NC-BCFR. 


\section{Empirical assessment of imputation accuracy}

We genotyped rs3778609, the top novel SNP, in the CAMA study in samples that also had GWAS data and checked the concordance between genotyped and imputed results.

\section{Genetic ancestry inference}

We implemented principal component (PC) analysis to assess genetic ancestry in each of the discovery datasets in unrelated individuals. To do so, we first LD-pruned typed SNPs with $r^{2}>0.2$ in PLINK. With the remaining data, we determined the PCs using EIGENSTRAT [47] within smartpca. For the replication datasets, we used ancestry informative markers and used the program ADMIXTURE [48] to calculate genetic ancestry, assuming a three-population model with ancestry from African, European, and Native American populations. We also inferred genetic ancestry as derived from the program ADMIXTURE in the discovery GWAS dataset to perform sensitivity analyses.

\section{Association testing}

We performed single-variant association testing using logistic regression models and adjusting for PCs 1-10 in PLINK [49]. For the replication datasets we entered ancestry into the model as covariates. For discovery, we performed GWAS by study and then performed a fixed effects meta-analysis using METAL [50]. We also performed association testing separately for estrogen receptor (ER)-positive and ER-negative breast cancer using this approach. To calculate LD, we calculated $R^{2}$ in the controls in our dataset using PLINK. We then performed conditional analyses by entering the most significant SNP in the model as a covariate in addition to PCs $1-10$. We evaluated genome-wide inflation by estimating $\lambda(\lambda \leq 1.0$ indicates no inflation). To test for heterogeneity with family history and study site we entered these as multiplicative interaction variables with the SNPs of interest in the logistic regression models and tested the significance of the interaction variables. To test for heterogeneity by age, we dichotomized at age 50 years and also tested for a multiplicative interaction with the SNPs. In addition, we re-tested the associations for the top SNPs adjusting for genetic ancestry from ADMIXTURE using logistic regression models. Heterogeneity analyses and the analysis of the top SNPs using ancestry estimates from ADMIXTURE were performed using Stata (Version 14).

\section{Power}

Based on the sample size for discovery (2385 cases and 6416 controls) we had $\sim 80 \%$ power to detect an odds ratio of $1.25,1.365$, and 1.49 with allele frequencies of $0.4,0.2$, and 0.1 respectively.

\section{Ranking SNPs by evidence for function}

For each of the top index SNPs (rs140068132, rs851985, and rs3776809) we identified all of the other SNPs that they are in LD with $\left(R^{2}>0.5\right)$ and that have a $p$ value that is at within $2 \log$ (base 10) level of significance compared to the top SNP. We then entered each index SNP with the other SNPs from that cluster into regulomeDB [51], which uses Encode data to annotate SNPs and report on their likelihood of affecting gene expression. The level of evidence could include the SNP being in a DNAse hypersensitivity region and/or a region associated with transcription factor (TF) binding. Further weight is given if the SNP alters a TF binding motif.

\section{Results}

Individual association analyses

We conducted a meta-analysis across four GWAS discovery studies (Table 1) and identified 48 variants with genome-wide significant $p$ values at the $6 \mathrm{q} 25$ susceptibility region (Additional file 1: Table S1). We saw no evidence for inflation of association statistics in genome-wide analysis $(\lambda=0.97)$. No additional genome-wide significant SNPs were identified.

Evaluating the SNPs at $6 \mathrm{q} 25$ and accounting for linkage disequilibrium (LD) between them, we found three distinct clusters of SNPs (haplotypes) associated at a genome-significant level (Fig. 1; Additional file 1:

Table 1 Discovery and replication samples used

\begin{tabular}{llll}
\hline Study & Genotyping platform & Cases & Controls \\
\hline Discovery: Latinas & & & \\
BCFR/SFBACS & Affy 6.0 & 942 & 589 \\
RPGEH & Affy Axiom & 225 & 3574 \\
MEC & Illumina 1 M, 2.5 M & 520 & 1544 \\
CAMA & Illumina Oncoarray & 698 & 599 \\
Total & & 2385 & 6416 \\
Replication: Latinas & & & \\
$\quad$ COLUMBUS & Affy Axiom & 954 & 769 \\
CCGCRN & Taqman & 1148 & 347 \\
CAMA & Taqman & 310 & 464 \\
Total Latina replication & & 2412 & 1580 \\
Replication: African American & & & \\
AABC & Illumina 1 M & 3153 & 2831 \\
ROOT & Illumina 2.5 M & 1657 & 2029 \\
Total African ancestry & & 4810 & 4860 \\
Replication: East Asian ancestry & & & \\
Shanghai Breast Cancer Study & Affymetrix 6.0 & 2731 & 2135 \\
Shanghai Breast Cancer Study & Illumina MEGA & 1794 & 2059 \\
Total East Asian population & & 4525 & 4194 \\
\hline
\end{tabular}


A

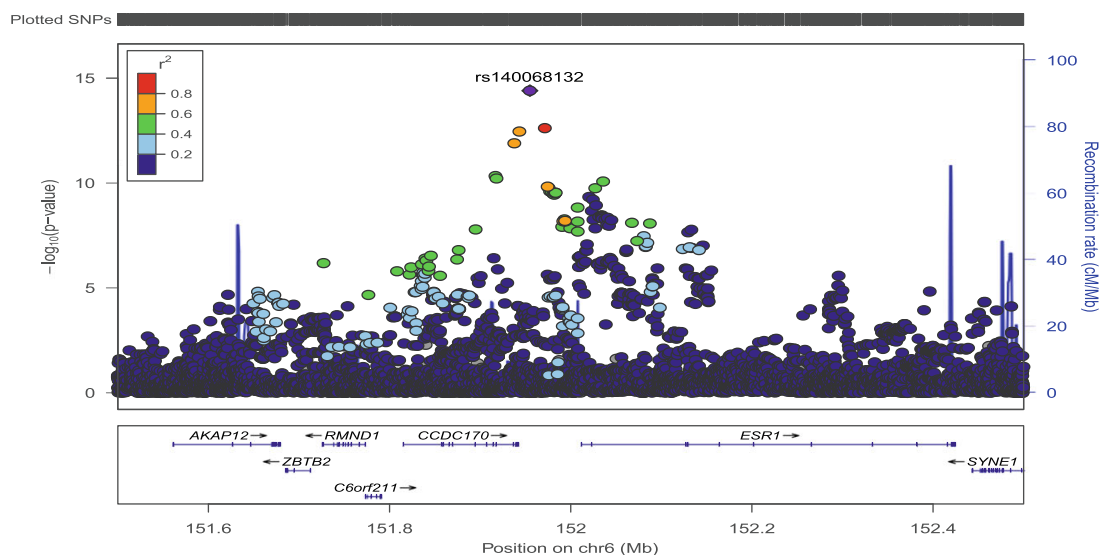

B

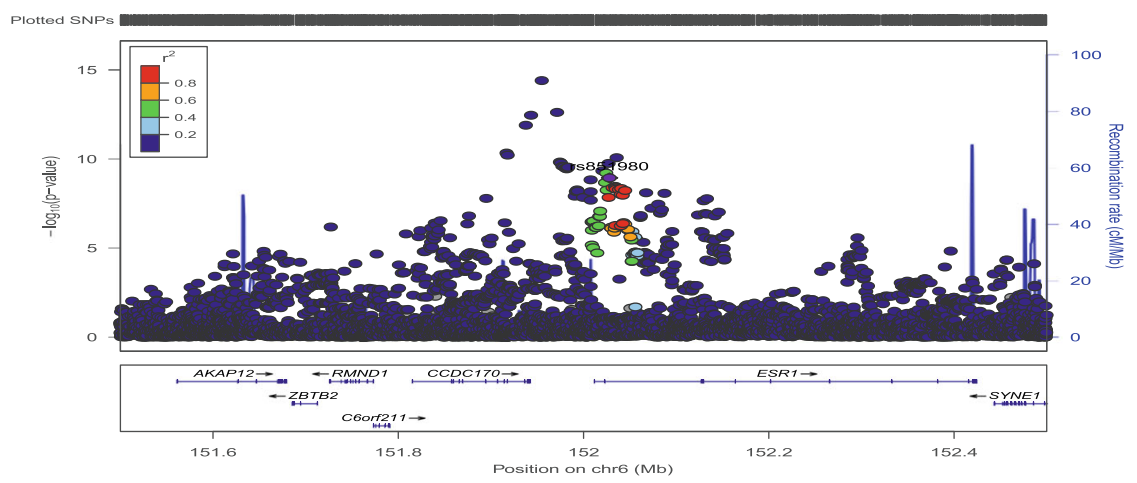

C

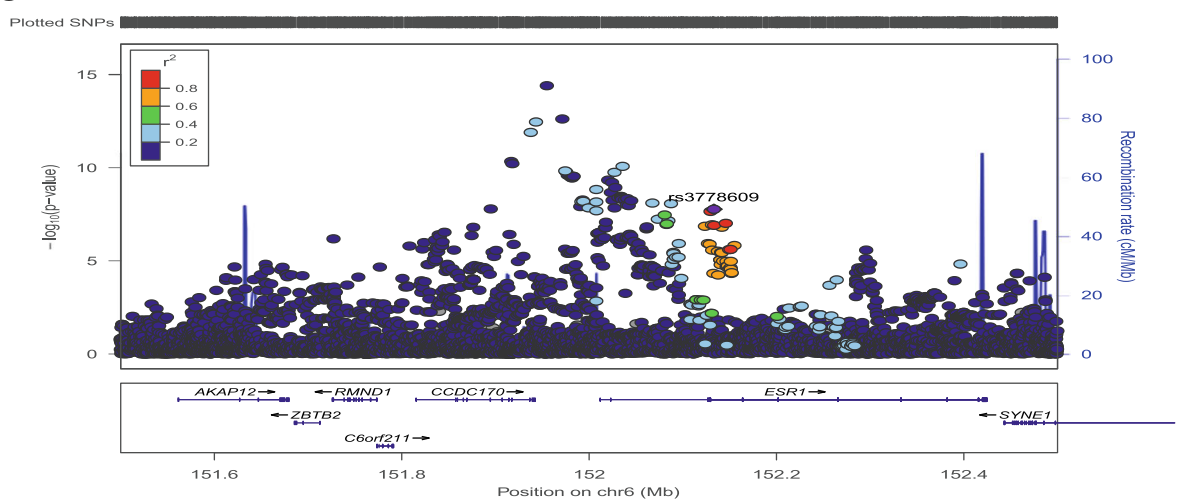

Fig. 1 Association results with SNPs on chromosome 6 (chr6) from position 151.5 megabases (Mb) to 152.5 MB. On the x-axis are chromosome positions and on the $y$-axis are the negative log (base 10) $p$ values. Each dot represents the meta-analysis results from the discovery genomewide association study (GWAS) datasets. The different panels represent coloring by different linkage disequilibrium (LD) values related to an index SNP. Three index SNPs are selected including rs1400685132 (a), rs851980 (b), and rs3778609 (c)

Table S1). The top variant in this region was rs140068132, which we previously reported as genomewide significant in this population [23]. Of the remaining 47 top variants, 21 were in strong $\left(r^{2}>0.5\right)$ and another six were in moderate $\left(0.39<r^{2}<0.49\right)$ LD with rs140068132, and none of these were significant in joint models with rs140068132 (Additional file 1: Table S1). Another set of 16 additional SNPs that was genome-wide significant, characterized by rs851980, were all in high LD $\left(r^{2}>0.5\right)$ with each other and in low LD $\left(r^{2}<0.2\right)$ with rs140068132. These SNPs include rs851984, which has been previously reported to be associated with breast 
cancer [27]. A third group of four SNPs at position 151.13-152.15 megabases (Mb) on chromosome 6 ( Hg19) was also genome-wide significant. These SNPs were characterized by rs3778609 and were in high LD $\left(r^{2}>0.9\right)$ with each and in low LD $\left(r^{2}<0.2\right)$ with rs140068132 or rs851985 and with other SNPs previously reported at this locus (Additional file 2: Table S2). The minor alleles of these SNPs are associated with lower risk of breast cancer, and the odds ratio (OR) for rs3778609 was 0.76 (95\% CI $0.69-0.83 ; p=6.0 \times 10^{-9}$; Table 2). These SNPs were well-imputed in each of the discovery GWAS with imputation $R^{2}$ values $>0.95$ for rs3778609 (Additional file 1: Table S1). Furthermore, in re-analysis of the top SNPs using 1000 Genomes as the reference dataset for imputation, we found a consistent genome-wide significant effect for these SNPs (Additional file 2: Table S3). We also evaluated the effect of controlling for genetic ancestry using a model-based approach (ADMIXTURE) in the discovery GWAS and found a consistent effect (Additional file 2: Table S4).

We performed conditional analyses by entering rs140068132 and other top SNPs at this locus in joint models. We found that rs3778609 remained nominally significant in a joint model adjusting for rs140068132 (Table 2), although the adjusted odds ratios were attenuated. We also found that rs851980 remained nominally significant in joint models with rs140068132 with mild attenuation. When we included three SNPs that best represent each of the signals from each set of associated variants (rs140068132, rs3778609, and rs851980) in the same model, all of the SNPs remained nominally significant with minimal attenuation of the odds ratios (compared to models including just pairs of variants; Table 2).

\section{Technical validation and replication}

We evaluated the imputation accuracy of rs3778609 in a sample of 1369 women from the CAMA study, which had imputed genotypes from the GWAS and genotypes determined by Taqman assay. We found excellent agreement between the imputed and genotyped data with $1361 / 1369$ (99.4\%) of the genotypes being in concordance.
We used data from the portion of the CAMA study that did not have GWAS data, the COLUMBUS study and the CCGCRN to replicate the association with rs3778609. We found a consistent direction in all three studies and a nominally significant association in a meta-analysis of the three studies $(N=2412 ; N=1620$; $\mathrm{OR}=0.88 ; 95 \%$ CI 0.78-0.99; $p=0.037$, Table 3 ).

\section{Association of previously identified SNPs at $6 q 25$}

We examined previously reported SNPs in our discovery dataset (Additional file 2: Table S5). Only rs851984 was genome-wide significant in our study. Two other SNPs, rs3757322 and rs9397437, were nominally significant in the correct direction. The other two were not significantly associated with breast cancer in our study but the effects were directionally consistent with the previous reports and the 95\% confidence intervals overlapped with the results from previous studies.

\section{Association with estrogen receptor subtypes and other sources of heterogeneity}

We analyzed the association for each of the top SNPs separately and jointly by ER-status (Table 4). As we have previously reported, the minor (low risk) allele of rs140068132 is associated with a significantly $(p=0.04)$ lower odds ratio for ER-negative than for ER-positive breast cancer. We also found a significantly stronger $(p=$ 0.025) effect size for ER-negative breast cancer for rs3778609. The effect size for rs851980 is also greater for ER-negative breast cancer; however, the difference between ER-negative and ER-positive breast cancer for this SNP was not statistically significant $(p=0.068)$.

We also evaluated for other sources of heterogeneity for rs3778609 including location (San Francisco Bay Area versus Los Angeles versus Mexico), age (dichotomizing at age 50 years) and family history. We found no evidence of heterogeneity with study site $(p=0.13)$, age $(p=0.71)$, or family history $(p=0.61)$.

\section{Replication in non-Latinas}

We evaluated the cluster of new SNPs we identified in this locus represented by rs37786109 in East Asians in the Shanghai breast cancer study and African ancestry populations in the ROOT and AABC studies and we also

Table 2 Representative SNPS and association statistics from each of four different SNP clusters/regions that are genome-wide significant

\begin{tabular}{lllllll}
\hline SNP/risk allele & Allele frequency & $\begin{array}{l}\text { Position } \\
(\mathrm{BP}, \mathrm{Hg} 19)\end{array}$ & $\begin{array}{l}\text { Odds ratio } \\
(95 \% \mathrm{Cl})\end{array}$ & $P$ value & $\begin{array}{l}\text { Conditional OR* } \\
P \text { value }\end{array}$ & $\begin{array}{l}\text {Joint OR }^{* *} \\
P \text { value }\end{array}$ \\
\hline rs140068132-G & 0.0933 & $6: 151954834$ & $0.58(0.50-0.66)$ & $4.4 \times 10^{-15}$ & & $0.61(0.53-0.71) 6.8 \times 10^{-12}$ \\
rs851980-C & 0.255 & $6: 152027955$ & $1.28(1.18-1.35)$ & $5.2 \times 10^{-10}$ & $1.22(1.12-1.32) 1.0 \times 10^{-6}$ & $1.19(1.10-1.28) 3 \times 10^{-6}$ \\
rs3778609-T & 0.192 & $6: 152133187$ & $0.76(0.69-0.83)$ & $6.0 \times 10^{-9}$ & $0.84(0.76-0.93) 6.5 \times 10^{-4}$ & $0.86(0.78-0.95) 0.0035$ \\
\hline
\end{tabular}

*Conditional on rs140068132

**Joint model with rs140068132, rs851985, and rs3778609 
Table 3 Replication of rs3778609 in other Latina datasets

\begin{tabular}{lll}
\hline Study & Odds ratio $(95 \% \mathrm{Cl})$ & $P$ value \\
\hline COLUMBUS & $0.87(0.73-1.04)$ & 0.119 \\
CCGCRN & $0.89(0.70-1.14)$ & 0.375 \\
CAMA (excluding GWAS) & $0.88(0.69-1.13)$ & 0.314 \\
Meta-analysis & $0.88(0.78-0.99)$ & 0.037 \\
\hline
\end{tabular}

GWAS genome-wide association studies

performed a look up of the effect in summary statistics from European populations (Table 5). We found a consistent effect for across these studies and a significant effect in a meta-analysis, although the odds ratio was a little attenuated compared to the odds ratio we observed in Latinas (Table 5).

\section{Evaluation of potential functional SNPs}

We evaluated the top SNPs from each of the three clusters of genome-wide significant SNPs at $6 \mathrm{q} 25$ to determine which has the strongest evidence of potentially functional effects on gene expression using regulomeDB [51]. Among the SNPs in LD with rs3778609, rs6914438 and rs2071454 had the best evidence of a functional effect (Additional file 3: Table S6). Rs691443 had positive results in MCF-7 cells for both DNAse hypersensitivity and transcription factor binding. Rs2071454 is in the 5' UTR of ESR1 and is also annotated as being in a DNAse hypersensitive region and a transcription factor binding site. At the two previously reported loci, rs140068132 and rs851984 had the best evidence for functional effect in regulomeDB.

\section{Discussion}

We have previously reported on a SNP at 6q25 associated with a minor allele that is unique to indigenous American populations and associated with decreased risk of breast cancer [23]. Here, we investigate this locus in greater depth in an expanded sample size of Latina breast cancer cases and controls. We have identified several SNPs that are genome-wide significant and associated with breast cancer at this locus independently of other SNPs previously reported by us in Latinas [23] and in other populations [11, 13, 24-27, 29, 31, 52, 53] at this locus. These SNPs are located in the region of 152.13-152.15 Mb (Hg19). Replication in African
American, Asian, and European samples supports the association with these SNPs. In addition, we have also shown that these novel variants at this locus have significantly stronger effect sizes on ER-negative breast cancer. Prior studies have also demonstrated a stronger effect size with ER-negative breast cancer for most variants at the $6 q 25$ locus, consistent with our data $[18,27]$.

Prior studies in other populations have reported a series of independent SNPs affecting breast cancer risk $[11,18,24-27,31]$. A comprehensive fine mapping project using European and Asian ancestry populations identified five different clusters of SNPs at this locus [27]. We found that one of these SNPs, rs851984, was also genome-wide significant in Latinas, two more were nominally significant, and the remaining two were non-significant but had effects that were directionally consistent with those observed by Dunning et al.

Dunning et al. found that the variants they identified affect expression of ESR1, RMND1 and $C C D C 170$ [27]. The ESR1 gene encodes estrogen receptor alpha, a strong candidate gene for an effect on breast cancer risk. The effect we detected was stronger for ER-negative breast cancer, consistent with the effect of most of the other SNPs reported at this locus. This differential effect is unexpected since estrogen signaling via ER is believed to increase the risk of ER-positive breast cancer and not have an effect on ER-negative breast cancer [54, 55]. However, ESR1 could also exert an effect via the stroma and many of the variants at this locus are known to affect mammographic density [27-30] which is associated with both ER-positive and ER-negative breast cancer [56]. Furthermore, other genes at this locus including ARMT1, CCDC170, and RMND1 may also be candidates for an effect on breast cancer risk [27, 57-59]. ARMT1 is protein methyl transferase that modifies DNA damage response [60] and small interfering RNA (siRNA) inhibition of ARMT1 by siRNA suppresses proliferation of MCF-7 cells [59]. CCDC170 is part of a recurrent rearrangement with ESR1 found in aggressive ER-positive breast cancers [61]. This rearrangement leads to a truncated $\mathrm{CCDC} 170$ protein which, when introduced into ER+ breast cancer cells, leads to increased cell motility, anchorage-independent growth, reduced endocrine sensitivity, and enhanced

Table 4 Association by estrogen receptor (ER) status

\begin{tabular}{|c|c|c|c|c|c|}
\hline \multirow[b]{2}{*}{ SNP } & \multicolumn{2}{|l|}{ ER-positive } & \multicolumn{2}{|l|}{ ER-negative } & \multirow{2}{*}{$\begin{array}{l}P \text { value for ER-positive } \\
\text { vs. ER-negative }\end{array}$} \\
\hline & Odds ratio $(95 \% \mathrm{Cl})$ & $P$ value & Odds ratio $(95 \% \mathrm{Cl})$ & $P$ value & \\
\hline rs140068132-G & $0.59(0.50-0.72)$ & $2.7 \times 10^{-7}$ & $0.39(0.27-0.55)$ & $2.0 \times 10^{-7}$ & 0.040 \\
\hline rs851980-C & $1.19(1.07-1.34)$ & 0.002 & $1.46(1.23-1.73)$ & $1.3 \times 10^{-5}$ & 0.068 \\
\hline rs3778609-T & $0.74(0.65-0.85)$ & $1.1 \times 10^{-5}$ & $0.59(0.47-0.73)$ & $2.0 \times 10^{-6}$ & 0.025 \\
\hline
\end{tabular}


Table 5 Associations of rs3778709 in non-Latina populations

\begin{tabular}{|c|c|c|c|c|c|}
\hline Study & Population & MAF & OR & SE & $P$ value \\
\hline$A A B C$ & African American & 0.304 & 0.93 & 0.044 & 0.10 \\
\hline ROOT & African, African American and West Indian & 0.323 & 0.96 & 0.052 & 0.48 \\
\hline Shanghai 1 & East Asian & 0.265 & 0.91 & 0.048 & 0.065 \\
\hline Shanghai 2 & East Asian & 0.264 & 0.99 & 0.052 & 0.84 \\
\hline European (Oncoarray + iCOGs) & European and European American & 0.016 & 0.94 & 0.025 & 0.025 \\
\hline Meta-analysis & & & 0.95 & 0.017 & 0.0019 \\
\hline
\end{tabular}

MAF minor allele frequency

xenograft tumor formation. Allele-specific expression studies suggest that breast cancer SNPs are more strongly associated with CCDC170 expression [57]. Since the new variants we report are common only in non-European ancestry populations, there are limited data to explore the potential effects of these variants on gene expression.

Our study is limited by sample size. Therefore, it is possible that we have missed other variants at this locus. Our study may also be affected by population stratification, which is more likely to occur in an admixed population such as Latinos. To reduce this problem, we adjusted for genetic ancestry in both the discovery and replication datasets. We found no evidence of genome-wide inflation of the association statistics in the discovery datasets. However, the replication datasets had fewer variants for the genetic ancestry estimates, which will lead to some noise in the ancestry estimate and may lead to insufficient adjustment. The studies we used included both population based and clinic-based recruitment and used different age ranges and, in some cases, family history criteria for ascertainment. Although we did not detect any interactions between the top new SNPs that we report and these factors, we may be underpowered to detect interactions.

We used the Haplotype Reference Consortium (HRC) for imputation, which includes a large number of reference individuals; however, the proportion of individuals with indigenous American ancestry in the HRC is low, and therefore, we are likely underpowered to detect rare variants that originate from American ancestry. The common variants that we report here are in the range that imputation from HRC is known to be relatively high quality. Furthermore, we validated our imputed results in one of the datasets by genotyping and found excellent correlation. Thus, we believe the new SNPs we report are based on relatively accurate imputation. However, we are likely missing some variants, particularly low-frequency variants from indigenous American origin. As imputation panels improve, we will be able to impute lower-frequency variants with more accuracy and may be able to detect new associations.

The effect size we observed in the replication dataset is substantially lower than in the discovery dataset, likely due to regression towards the mean, a well-known phenomenon in GWAS, since the top SNPs in a discovery dataset are likely to have some upward bias in the effect size [62]. However, even if we take the replication odds ratio $(0.88)$ as the closest to the true effect size of these SNPs, this is still a relatively large effect for a common variant. We also found a consistent (though further attenuated) effect in populations of African, Asian, and European ancestry. The attenuated effect size in these populations may be due to different LD patterns or may be due to other non-genetic modifiers. Of note, despite the very large sample size used for discovery in Europeans, this variant had not reached genome-wide significance due to the low allele frequency in Europeans. This highlights the utility of using different ancestry populations including Latinas, to help discover new variants. It is likely that there are other variants that have not yet been identified in European GWAS due to low allele frequency and that could be identified in Latinas where they are more common. Larger studies of Latina women are needed to identify these variants.

\section{Conclusion}

Our study demonstrates additional unique associations with variants at $6 \mathrm{q} 25$ and breast cancer risk. This further highlights the important contribution of this locus to breast cancer susceptibility, particularly ER-negative breast cancer susceptibility. Additional fine-mapping and functional studies are needed to elucidate all of the causal variants in our population. However, the variants we identified in this study can be useful to add to the increasing pool of common variants coming from GWAS and will be particularly useful to risk-stratify women of Latin American ancestry for breast cancer risk. 


\section{Additional files}

Additional file 1: Table S1. Association statistics and imputation accuracy for all SNPs with $p$ value $<5 \times 10-7$ in discovery dataset. Also included are results for models that are conditional on top SNPs and the and linkage disequilibrium between index SNPs described in the manuscript and other SNPs in this list. (XLSX $29 \mathrm{~kb}$ )

Additional file 2: Tables S2-S5. Supplementary tables including linkage disequilibrium between top SNPS and previously described SNPS at this locus. Association results for top SNPs with imputation to 1000 Genomes. Association results for top SNPs adjusted for ancestry using ADMIXTURE results. Association results for SNPs previously reported as genome wide significant at 6q25. (DOCX $27 \mathrm{~kb}$ )

Additional file 3: Table S6. Results of the top candidate SNPS analyzed using RegulomeDB (XLSX $12 \mathrm{~kb}$ )

\section{Abbreviations}

AABC: African American breast cancer study; BCFR: Breast Cancer Family Registry; CAMA: Cancer de mama; CARE: Women's contraceptive and reproductive experiences; CBCS: Carolina breast cancer study: CCGCRN: Clinical Cancer Genetics Community Research Network; $\mathrm{Cl}$ : Confidence interval; COLUMBUS: Colombian study of environmental and heritable causes of breast cancer; ER: Estrogen receptor; GWAS: Genomewide association study; HCRCOH: Hereditary cancer registry of City of Hope; IRB: Institutional review board; LD: Linkage disequilibrium; MAF: Minor allele frequency; Mb: Megabases; MEC: Multiethnic cohort; NC-BCFR: Northern California Breast Cancer Family Registry; OR: Odds ratio; PC: Principal component; PCA: Principal components analysis; RPGEH: Research project on genes environment and health; SBCS: Shanghai breast cancer study; SBCSS: Shanghai breast cancer survival study; SCCS: Southern community cohort study; SFBCS: San Francisco Bay Area breast cancer study; SNP: Single nucleotide polymorphism; SWHS: Shanghai women's health study; UTR: Untranslated region; WCHS: Women's circle of health study

\section{Acknowledgements}

The authors are grateful to study staff who recruited participants and to the participants who donated their samples and their time to these studies.

\section{Funding}

This work was funded in part by grants from the National Cancer Institute (K24CA169004, R01CA120120 to E Ziv and R01CA184545 to E Ziv and S Neuhausen). J Hoffman was supported by R25CA112355. The San Francisco Bay Area Breast Cancer Study was supported by the National Cancer Institute (CA63446 and CA77305), the US Department of Defense (DAMD17-96-16071) and the California Breast Cancer Research Program (7 PB-0068). The Northern California Breast Cancer Family Registry was supported by grant UM1 CA164920 from the National Cancer Institute. The Multiethnic Cohort Study was supported by the National Institutes of Health grants R01 CA63464 and R37 CA54281, R01 CA132839, 5UM1CA164973. The CAMA Study was funded by Consejo Nacional de Ciencia y Tecnología (SALUD2002-C01-7462). The COLUMBUS study receives support from GSK Oncology (Ethnic Research Initiative), University of Tolima, University of California Davis and The V Foundation. L.C.-C. is a V Foundation V Scholar. The Shanghai study was supported by R01CA148667 and UM1 CA182910. The ROOT study was supported by the American Cancer Society (MRSG-13-063-01-TBG and CRP-10-119-01-CCE), National Cancer Institute (CA142996, CA161032), Susan G. Komen for the Cure, and Breast Cancer Research Foundation. The GALA study was supported by the Sandler Family Foundation, the American Asthma Foundation, the RWJF Amos Medical Faculty Development Program, Harry Wm. and Diana V. Hind Distinguished Professor in Pharmaceutical Sciences II, National Institutes of Health 1R01HL117004, R01HI128439,

R01HL135156, 1X01HL134589, National Institute of Health and Environmental Health Sciences R01ES015794, R21ES24844, the National Institute on Minority Health and Health Disparities 1P60MD006902, RL5GM118984, 1R01MD010443 and the Tobacco-Related Disease Research Program under Award Number 24RT-0025.

\section{Availability of data and materials}

The following GWAS datasets have been submitted to dbGAP: SFBACS (phs000912.v1.p1), MEC Latina (phs000517.v2.p1), AABC (phs000851.v1.p1), ROOT (phs000383.v1.p1), Shanghai Breast Cancer Study (phs000799.v1.p1). The NC-BCFR was determined by the IRB not to be eligible for submission to dbGAP based on the consent. Summary statistics are available for the $6 \mathrm{q} 25$ locus from the NC-BCFR, the Kaiser RPGEH, and the COLUMBUS study and are available from the corresponding author by request.

\section{Authors' contributions}

$J H, L F$, and EZ conceived of the study. JH, LF, DH, SH, and EZ conducted statistical analyses including imputation, GWAS, and replication analyses. ML and YCD conducted replication genotyping. $\mathrm{CH}$ contributed samples and data, contributed to analyses, and made critical comments on the manuscript. EJ, GTM, LK, SN, JW, OO, DH, JL, WZ, PL ME LCC, EB, and CE contributed samples and data and made revisions to the manuscript. All authors read and approved the final manuscript.

\section{Ethics approval and consent to participate}

The following Institutional Review Boards (IRBs) have approved the collection of different datasets, which have contributed to this study: University of California, San Francisco, the Cancer Prevention Institute of California, University of Southern California, Kaiser Permanente Division of Research Northern California, University of Chicago, Vanderbilt University, National Institute of Public Health, Mexico.

\section{Consent for publication}

Not applicable.

\section{Competing interests}

The authors declare that they have no competing interests.

\section{Publisher's Note}

Springer Nature remains neutral with regard to jurisdictional claims in published maps and institutional affiliations.

\section{Author details}

'Department of Epidemiology and Biostatistics, Institute of Human Genetics, University of California, San Francisco, San Francisco, CA, USA. ${ }^{2}$ Division of General Internal Medicine, Department of Medicine, Institute of Human Genetics, Helen Diller Family Comprehensive Cancer Center, University of California, San Francisco, Box 0320, San Francisco, CA 94143, USA. ${ }^{3}$ Division of General Internal Medicine, Department of Medicine, University of California, San Francisco, San Francisco, CA, USA. ${ }^{4}$ Department of Medicine, Stanford University, Stanford, CA, USA. ${ }^{5}$ Stanford Cancer Institute, Stanford University, Stanford, CA, USA. ${ }^{6}$ National Institute of Public Health, Cuernavaca, Mexico. 'Division of Research, Kaiser Permanente, Oakland, Northern California, USA. ${ }^{8}$ Beckman Research Institute of City of Hope, Department of Population Sciences, City of Hope, Duarte, CA, USA. ${ }^{9}$ City of Hope National Medical Center, Clinical Cancer Genetics, Duarte, CA, USA. ${ }^{10}$ Department of Biochemistry and Molecular Medicine, University of California, Davis, Davis, CA, USA. ${ }^{11}$ University of Tolima, Ibague, Colombia. ${ }^{12}$ Department of Biophamaceutical Sciences, Lung Biology Center, University of California, San Francisco, San Francisco, CA, USA. ${ }^{13}$ Department of Medicine, Institute for Human Genetics, University of California, San Francisco, San Francisco, CA, USA. ${ }^{14}$ Department of Epidemiology, Vanderbilt University Medical Center, Nashville, TN, USA. ${ }^{15}$ Department of Public Health Sciences, University of Chicago School of Medicine, Chicago, IL, USA. ${ }^{16}$ Department of Medicine, Section of Oncology, University of Chicago School of Medicine, Chicago, IL, USA. ${ }^{17}$ Department of Preventive Medicine, Norris Comprehensive Cancer Center, Keck School of Medicine, University of Southern California, Los Angeles, CA, USA.

Received: 9 April 2018 Accepted: 4 December 2018

Published online: 14 January 2019

\section{References}

1. Hall JM, Lee MK, Newman B, Morrow JE, Anderson LA, Huey B, King MC. Linkage of early-onset familial breast cancer to chromosome 17q21. Science. 1990;250(4988):1684-9. 
2. Miki Y, Swensen J, Shattuck-Eidens D, Futreal PA, Harshman K, Tavtigian S, Liu Q, Cochran C, Bennett LM, Ding W, et al. A strong candidate for the breast and ovarian cancer susceptibility gene BRCA1. Science. 1994; 266(5182):66-71.

3. Wooster R, Bignell G, Lancaster J, Swift S, Seal S, Mangion J, Collins N, Gregory S, Gumbs C, Micklem G. Identification of the breast cancer susceptibility gene BRCA2. Nature. 1995;378(6559):789-92.

4. Walsh T, King MC. Ten genes for inherited breast cancer. Cancer Cell. 2007; 11(2):103-5

5. Easton DF, Pooley KA, Dunning AM, Pharoah PD, Thompson D, Ballinger DG, Struewing JP, Morrison J, Field H, Luben R, et al. Genome-wide association study identifies novel breast cancer susceptibility loci. Nature. 2007; 447(7148):1087-93.

6. Hunter DJ, Kraft P, Jacobs KB, Cox DG, Yeager M, Hankinson SE, Wacholder S, Wang Z, Welch R, Hutchinson A, et al. A genome-wide association study identifies alleles in FGFR2 associated with risk of sporadic postmenopausal breast cancer. Nat Genet. 2007;39(7):870-4.

7. Stacey SN, Manolescu A, Sulem P, Rafnar T, Gudmundsson J, Gudjonsson SA, Masson G, Jakobsdottir M, Thorlacius S, Helgason A, et al. Common variants on chromosomes $2 \mathrm{q} 35$ and $16 \mathrm{q} 12$ confer susceptibility to estrogen receptor-positive breast cancer. Nat Genet. 2007:39(7):865-9.

8. Ahmed S, Thomas G, Ghoussaini M, Healey CS, Humphreys MK, Platte R, Morrison J, Maranian M, Pooley KA, Luben R, et al. Newly discovered breast cancer susceptibility loci on 3p24 and 17q23.2. Nat Genet. 2009;41(5):585-90.

9. Thomas G, Jacobs KB, Kraft P, Yeager M, Wacholder S, Cox DG, Hankinson SE, Hutchinson A, Wang Z, Yu K, et al. A multistage genome-wide association study in breast cancer identifies two new risk alleles at 1 p11.2 and 14q24.1 (RAD51L1). Nat Genet. 2009;41(5):579-84.

10. Long J, Cai Q, Shu XO, Qu S, Li C, Zheng Y, Gu K, Wang W, Xiang YB, Cheng $J$, et al. Identification of a functional genetic variant at $16 q 12.1$ for breast cancer risk: results from the Asia Breast Cancer Consortium. PLoS Genet. 2010;6(6):e1001002.

11. Turnbull C, Ahmed S, Morrison J, Pernet D, Renwick A, Maranian M, Seal S, Ghoussaini M, Hines S, Healey CS, et al. Genome-wide association study identifies five new breast cancer susceptibility loci. Nat Genet. 2010;42(6):504-7.

12. Wang X, Pankratz VS, Fredericksen Z, Tarrell R, Karaus M, McGuffog L, Pharaoh PD, Ponder BA, Dunning AM, Peock S, et al. Common variants associated with breast cancer in genome-wide association studies are modifiers of breast cancer risk in BRCA1 and BRCA2 mutation carriers. Hum Mol Genet. 2010;19(14):2886-97.

13. Antoniou AC, Kartsonaki C, Sinilnikova OM, Soucy P, McGuffog L, Healey S, Lee A, Peterlongo P, Manoukian S, Peissel B, et al. Common alleles at 6q25.1 and 1 p11.2 are associated with breast cancer risk for BRCA1 and BRCA2 mutation carriers. Hum Mol Genet. 2011;20(16):3304-21.

14. Cai Q, Long J, Lu W, Qu S, Wen W, Kang D, Lee JY, Chen K, Shen H, Shen $C Y$, et al. Genome-wide association study identifies breast cancer risk variant at 10q21.2: results from the Asia Breast Cancer Consortium. Hum Mol Genet. 2011;20(24):4991-9.

15. Haiman CA, Chen GK, Vachon CM, Canzian F, Dunning A, Millikan RC, Wang $X$, Ademuyiwa F, Ahmed S, Ambrosone CB, et al. A common variant at the TERT-CLPTM1L locus is associated with estrogen receptor-negative breast cancer. Nat Genet. 2011;43(12):1210-4.

16. Couch FJ, Gaudet MM, Antoniou AC, Ramus SJ, Kuchenbaecker KB, Soucy P, Beesley J, Chen X, Wang X, Kirchhoff T, et al. Common variants at the 19p13.1 and ZNF365 loci are associated with ER subtypes of breast cancer and ovarian cancer risk in BRCA1 and BRCA2 mutation carriers. Cancer Epidemiol Biomark Prev. 2012;21(4):645-57.

17. Ghoussaini M, Fletcher O, Michailidou K, Turnbull C, Schmidt MK, Dicks E, Dennis J, Wang Q, Humphreys MK, Luccarini C, et al. Genome-wide association analysis identifies three new breast cancer susceptibility loci. Nat Genet. 2012;44(3):312-8.

18. Siddiq A, Couch FJ, Chen GK, Lindstrom S, Eccles D, Millikan RC, Michailidou K, Stram DO, Beckmann L, Rhie SK, et al. A meta-analysis of genome-wide association studies of breast cancer identifies two novel susceptibility loci at $6 q 14$ and 20q11. Hum Mol Genet. 2012;21(24):5373-84.

19. Michailidou K, Hall P, Gonzalez-Neira A, Ghoussaini M, Dennis J, Milne RL, Schmidt MK, Chang-Claude J, Bojesen SE, Bolla MK, et al. Large-scale genotyping identifies 41 new loci associated with breast cancer risk. Nat Genet. 2013;45(4):353-61 361e351-352.

20. Zheng W, Zhang B, Cai Q, Sung H, Michailidou K, Shi J, Choi JY, Long J, Dennis J, Humphreys MK, et al. Common genetic determinants of breast- cancer risk in East Asian women: a collaborative study of 23637 breast cancer cases and 25579 controls. Hum Mol Genet. 2013;22(12):2539-50.

21. Huo D, Feng Y, Haddad S, Zheng Y, Yao S, Han YJ, Ogundiran TO, Adebamowo C, Ojengbede O, Falusi AG, et al. Genome-wide association studies in women of African ancestry identified 3q26.21 as a novel susceptibility locus for oestrogen receptor negative breast cancer. Hum Mol Genet. 2016;25(21):4835-46.

22. Fejerman L, Chen GK, Eng C, Huntsman S, Hu D, Williams A, Pasaniuc B, John EM, Via M, Gignoux C, et al. Admixture mapping identifies a locus on 6 q25 associated with breast cancer risk in US Latinas. Hum Mol Genet. 2012; 21(8):1907-17.

23. Fejerman L, Ahmadiyeh N, Hu D, Huntsman S, Beckman KB, Caswell JL, Tsung K, John EM, Torres-Mejia G, Carvajal-Carmona L, et al. Genome-wide association study of breast cancer in Latinas identifies novel protective variants on 6q25. Nat Commun. 2014:5:5260.

24. Zheng W, Long J, Gao YT, Li C, Zheng Y, Xiang YB, Wen W, Levy S, Deming $\mathrm{SL}$, Haines $J$, et al. Genome-wide association study identifies a new breast cancer susceptibility locus at 6q25.1. Nat Genet. 2009;41(3):324-8.

25. Cai Q, Wen W, Qu S, Li G, Egan KM, Chen K, Deming SL, Shen H, Shen CY, Gammon MD, et al. Replication and functional genomic analyses of the breast cancer susceptibility locus at 6q25.1 generalize its importance in women of chinese, Japanese, and European ancestry. Cancer Res. 2011; 71(4):1344-55.

26. Hein R, Maranian M, Hopper JL, Kapuscinski MK, Southey MC, Park DJ, Schmidt MK, Broeks A, Hogervorst FB, Bueno-de-Mesquita HB, et al. Comparison of $6 \mathrm{q} 25$ breast cancer hits from Asian and European genome wide association studies in the Breast Cancer Association Consortium (BCAC). PLoS One. 2012;7(8):e42380.

27. Dunning AM, Michailidou K, Kuchenbaecker KB, Thompson D, French JD, Beesley J, Healey CS, Kar S, Pooley KA, Lopez-Knowles E, et al. Breast cancer risk variants at $6 \mathrm{q} 25$ display different phenotype associations and regulate ESR1, RMND1 and CCDC170. Nat Genet. 2016:48(4):374-86.

28. Brand JS, Li J, Humphreys K, Karlsson R, Eriksson M, Ivansson E, Hall P, Czene K. Identification of two novel mammographic density loci at 6Q25.1. Breast Cancer Res. 2015;17:75.

29. Ellingjord-Dale M, Grotmol T, Lee E, Van Den Berg DJ, Hofvind S, Couto E, Sovio U, Dos-Santos-Silva I, Ursin G. Breast cancer susceptibility variants and mammographic density phenotypes in norwegian postmenopausal women. Cancer Epidemiol Biomark Prev. 2014;23(9):1752-63.

30. Mariapun S, Ho WK, Kang PC, Li J, Lindstrom S, Yip CH, Teo SH. Variants in 6q25.1 are associated with mammographic density in Malaysian Chinese women. Cancer Epidemiol Biomark Prev. 2016;25(2):327-33.

31. Long J, Cai Q, Sung H, Shi J, Zhang B, Choi JY, Wen W, Delahanty RJ, Lu W, Gao YT, et al. Genome-wide association study in east Asians identifies novel susceptibility loci for breast cancer. PLoS Genet. 2012;8(2):e1002532.

32. Gonzalez Burchard E, Borrell LN, Choudhry S, Naqvi M, Tsai HJ, Rodriguez-Santana JR, Chapela R, Rogers SD, Mei R, Rodriguez-Cintron W, et al. Latino populations: a unique opportunity for the study of race, genetics, and social environment in epidemiological research. Am J Public Health. 2005;95(12):2161-8.

33. Park SL, Cheng I, Haiman CA. Genome-wide association studies of cancer in diverse populations. Cancer Epidemiol Biomarkers Prev. 2018; 27(4):405-17.

34. Lisker R, Ramirez E, Briceno RP, Granados J, Babinsky V. Gene frequencies and admixture estimates in four Mexican urban centers. Hum Biol. 1990; 62(6):791-801.

35. Hanis CL, Hewett-Emmett D, Bertin TK, Schull WJ. Origins of U.S. Hispanics. Implications for diabetes. Diabetes Care. 1991;14(7):618-27.

36. Williams RC, Knowler WC, Pettitt DJ, Long JC, Rokala DA, Polesky HF, Hackenberg RA, Steinberg AG, Bennett PH. The magnitude and origin of European-American admixture in the Gila River Indian Community of Arizona: a union of genetics and demography. Am J Hum Genet. 1992; 51(1):101-10.

37. Bertoni B, Budowle B, Sans M, Barton SA, Chakraborty R. Admixture in Hispanics: distribution of ancestral population contributions in the Continental United States. Hum Biol. 2003;75(1):1-11.

38. MacDonald DJ, Blazer KR, Weitzel JN. Extending comprehensive cancer center expertise in clinical cancer genetics and genomics to diverse communities: the power of partnership. J Natl Compr Canc Netw. 2010; $8(5): 615-24$

39. Feng $Y$, Stram DO, Rhie SK, Millikan RC, Ambrosone CB, John EM, Bernstein $\mathrm{L}$, Zheng W, Olshan AF, Hu J, et al. A comprehensive examination of breast 
cancer risk loci in African American women. Hum Mol Genet. 2014;23(20): 5518-26.

40. Michailidou K, Lindstrom S, Dennis J, Beesley J, Hui S, Kar S, Lemacon A, Soucy P, Glubb D, Rostamianfar A, et al. Association analysis identifies 65 new breast cancer risk loci. Nature. 2017;551(7678):92-4.

41. Fejerman L, Romieu I, John EM, Lazcano-Ponce E, Huntsman S, Beckman KB, Perez-Stable EJ, Gonzalez Burchard E, Ziv E, Torres-Mejia G. European ancestry is positively associated with breast cancer risk in Mexican women. Cancer Epidemiol Biomark Prev. 2010;19(4):1074-82.

42. DePristo MA, Banks E, Poplin R, Garimella KV, Maguire JR, Hartl C, Philippakis AA, del Angel G, Rivas MA, Hanna M, et al. A framework for variation discovery and genotyping using next-generation DNA sequencing data. Nat Genet. 2011;43(5):491-8.

43. Genomes Project C, Abecasis GR, Auton A, Brooks LD, MA DP, Durbin RM, Handsaker RE, Kang HM, Marth GT, McVean GA. An integrated map of genetic variation from 1,092 human genomes. Nature. 2012;491(7422):56-65.

44. Genomes Project C, Auton A, Brooks LD, Durbin RM, Garrison EP, Kang HM, Korbel JO, Marchini JL, McCarthy S, McVean GA, et al. A global reference for human genetic variation. Nature. 2015;526(7571):68-74.

45. Howie BN, Donnelly P, Marchini J. A flexible and accurate genotype imputation method for the next generation of genome-wide association studies. PLoS Genet. 2009;5(6):e1000529.

46. Manichaikul A, Mychaleckyj JC, Rich SS, Daly K, Sale M, Chen WM. Robust relationship inference in genome-wide association studies. Bioinformatics. 2010;26(22):2867-73

47. Price AL, Patterson NJ, Plenge RM, Weinblatt ME, Shadick NA, Reich D. Principal components analysis corrects for stratification in genome-wide association studies. Nat Genet. 2006;38(8):904-9.

48. Alexander DH, Novembre J, Lange K. Fast model-based estimation of ancestry in unrelated individuals. Genome Res. 2009;19(9):1655-64.

49. Purcell S, Neale B, Todd-Brown K, Thomas L, Ferreira MA, Bender D, Maller J, Sklar P, de Bakker PI, Daly MJ, et al. PLINK: a tool set for whole-genome association and population-based linkage analyses. Am J Hum Genet. 2007; 81(3):559-75.

50. Willer CJ, Li Y, Abecasis GR. METAL: fast and efficient meta-analysis of genomewide association scans. Bioinformatics. 2010;26(17):2190-1.

51. Boyle AP, Hong EL, Hariharan M, Cheng Y, Schaub MA, Kasowski M, Karczewski $\mathrm{KJ}$, Park J, Hitz BC, Weng S, et al. Annotation of functional variation in personal genomes using RegulomeDB. Genome Res. 2012;22(9):1790-7.

52. Long J, Zhang B, Signorello LB, Cai Q, Deming-Halverson S, Shrubsole MJ, Sanderson M, Dennis J, Michailidou K, Easton DF, et al. Evaluating genomewide association study-identified breast cancer risk variants in AfricanAmerican women. PLoS One. 2013;8(4):e58350.

53. Stacey SN, Sulem P, Zanon C, Gudjonsson SA, Thorleifsson G, Helgason A, Jonasdottir A, Besenbacher S, Kostic JP, Fackenthal JD, et al. Ancestry-shift refinement mapping of the C6orf97-ESR1 breast cancer susceptibility locus. PLoS Genet. 2010;6(7):e1001029.

54. Cummings SR, Lee JS, Lui LY, Stone K, Ljung BM, Cauleys JA. Sex hormones, risk factors, and risk of estrogen receptor-positive breast cancer in older women: a long-term prospective study. Cancer Epidemiol Biomark Prev. 2005;14(5):1047-51.

55. Cummings SR, Eckert S, Krueger KA, Grady D, Powles TJ, Cauley JA, Norton L, Nickelsen T, Bjarnason NH, Morrow M, et al. The effect of raloxifene on risk of breast cancer in postmenopausal women: results from the MORE randomized trial. Multiple Outcomes of Raloxifene Evaluation. JAMA. 1999; 281(23):2189-97.

56. Ziv E, Tice J, Smith-Bindman R, Shepherd J, Cummings S, Kerlikowske K. Mammographic density and estrogen receptor status of breast cancer. Cancer Epidemiol Biomark Prev. 2004;13(12):2090-5.

57. Jiang P, Li Y, Poleshko A, Medvedeva V, Baulina N, Zhang Y, Zhou Y, Slater CM, Pellegrin T, Wasserman J, et al. The protein encoded by the CCDC170 breast cancer gene functions to organize the golgi-microtubule network. EBioMedicine. 2017;22:28-43.

58. Yamamoto-lbusuki M, Yamamoto Y, Fujiwara S, Sueta A, Yamamoto S, Hayashi M, Tomiguchi M, Takeshita T, Iwase H. C6ORF97-ESR1 breast cancer susceptibility locus: influence on progression and survival in breast cancer patients. Eur J Hum Genet. 2015;23(7):949-56.

59. Dunbier AK, Anderson H, Ghazoui Z, Lopez-Knowles E, Pancholi S, Ribas R, Drury S, Sidhu K, Leary A, Martin LA, et al. ESR1 is co-expressed with closely adjacent uncharacterised genes spanning a breast cancer susceptibility locus at 6q25.1. PLoS Genet. 2011;7(4):e1001382.
60. Perry JJ, Ballard GD, Albert AE, Dobrolecki LE, Malkas LH, Hoelz DJ. Human C6orf211 encodes Armt1, a protein carboxyl methyltransferase that targets PCNA and is linked to the DNA damage response. Cell Rep. 2015;10(8): 1288-96.

61. Veeraraghavan J, Tan Y, Cao XX, Kim JA, Wang X, Chamness GC, Maiti SN, Cooper L, Edwards DP, Contreras A, et al. Recurrent ESR1-CCDC170 rearrangements in an aggressive subset of oestrogen receptor-positive breast cancers. Nat Commun. 2014;5:4577.

62. Xiao R, Boehnke M. Quantifying and correcting for the winner's curse in genetic association studies. Genet Epidemiol. 2009;33(5):453-62.
Ready to submit your research? Choose BMC and benefit from:

- fast, convenient online submission

- thorough peer review by experienced researchers in your field

- rapid publication on acceptance

- support for research data, including large and complex data types

- gold Open Access which fosters wider collaboration and increased citations

- maximum visibility for your research: over $100 \mathrm{M}$ website views per year

At BMC, research is always in progress.

Learn more biomedcentral.com/submissions 